\title{
Testing for fecal gluten immunogenic peptides: a useful tool to evaluate compliance with gluten-free diet by celiacs
}

\section{Brunetta Porcellia, Fabio Ferretti ${ }^{b}$, Ivano Biviano ${ }^{c}$, Alessia Santinic, Francesca Cincia ${ }^{a}$, Marina Vascotto ${ }^{d}$, Elisabetta Grande d, Francesco Quagliarellad, Lucia Terzuolia, Nicola Bizzaroe, Mario Marinic, Silvia Rentinic}

Università degli Studi di Siena; Azienda Ospedaliera Universitaria Senese, Siena, Azienda Sanitaria Universitaria Integrata di Udine, Tolmezzo, Italy

\section{Abstract}

\begin{abstract}
Background Although experts agree that strict dietary compliance is fundamental for the health of celiac patients, there are no evidence-based recommendations on the best way to assess dietary compliance. Detection of gluten immunogenic peptides (GIPs) in feces was recently proposed as an effective method of assessing the dietary compliance of celiac patients.
\end{abstract}

Methods Fifty-five consecutive celiac patients ( 27 adults and 28 children, age 6-72 years), who had been on a gluten-free diet for at least 2 years, were enrolled. All patients were evaluated clinically for symptoms, physical parameters and laboratory parameters. Dietary compliance was assessed with the Biagi questionnaire and serum anti-tissue transglutaminase (tTG) IgA antibodies were measured. GIPs were determined by immunoenzymatic assay on an automated Chorus analyzer (DIESSE Diagnostica Senese), after extraction of fecal samples by the method developed by DIESSE.

Results Eight patients tested positive for GIPs (GIPs+); 71.4\% of GIP-positive patients were asymptomatic; tTG antibodies were detected in $3 / 8 \mathrm{GIP}+$ patients. The Biagi score was significantly associated with fecal positivity for GIPs $(\mathrm{P}=0.02)$. However, according to the Biagi score, $57.1 \%$ of GIP+ patients followed the diet strictly and $5.4 \%$ of GIP- subjects did not comply with the diet or made substantial mistakes.

Conclusions Assay of fecal GIPs identified more patients who did not comply with the diet than did the Biagi questionnaire, evaluation of symptoms or anti-tTG antibodies. Detection of fecal GIPs offers a direct, objective, quantitative assessment of even occasional exposure to gluten and is confirmed as a practical way to check dietary compliance.

Keywords Fecal gluten peptides, gluten-free diet, celiac disease

Ann Gastroenterol 2020; 33 (6): 1-7
aSezione di Biochimica, Dipartimento Biotecnologie Mediche, Università degli Studi di Siena (Brunetta Porcelli, Francesca Cinci, Lucia Terzuoli); ${ }^{b}$ Dipartimento Scienze Mediche, Chirurgiche e Neuroscienze, Università degli Studi di Siena (Fabio Ferretti); 'UOC Gastroenterologia ed Endoscopia Operativa, Azienda Ospedaliera Universitaria Senese (Ivano Biviano, Alessia Santini, Mario Marini, Silvia Rentini); ${ }^{\mathrm{d} U O C}$ Pediatria, Azienda Ospedaliera Universitaria Senese, Siena (Marina Vascotto, Elisabetta Grande, Francesco Quagliarella); ${ }^{\mathrm{e}}$ Laboratorio di Patologia Clinica, Ospedale San Antonio, Azienda Sanitaria Universitaria Integrata di Udine, Tolmezzo (Nicola Bizzaro), Italy

\section{Conflict of Interest: None}

Correspondence to: Prof. Brunetta Porcelli, Dipartimento Biotecnologie Mediche, Sezione di Biochimica, Polo Scientifico Universitario di San Miniato, Via Alcide de Gasperi 2, 53100 Siena, Italy, e-mail: brunetta.porcelli@unisi.it

Received 10 May 2020; accepted 29 June 2020; published online 16 September 2020

DOI: https://doi.org/10.20524/aog.2020.0530

\section{Introduction}

Celiac disease (CD) is an immune-mediated pathology caused by dietary intake of gluten, which in genetically predisposed subjects causes a chronic inflammatory reaction of the small intestinal mucosa, leading to malabsorption due to gradual flattening of mucosa cells and loss of intestinal villi [1]. Gluten is a protein aggregate of heterogeneous composition found in wheat and other cereals such as barley and rye. It is largely composed of gliadin and glutenin, with a high proline and glutamine content that enable it to resist proteolytic breakdown in the gastrointestinal tract, leading to the formation of various gluten peptides during digestion. Some of these peptides are strongly immunogenic. In particular, a peptide derived from $\alpha-2$ gliadin, 33-mer, shows a high capacity to activate CD4+ T lymphocytes and has been identified as the main cause of gluten immunotoxicity [2]. 
Currently a strict lifelong gluten-free diet (GFD) is the only available treatment that can ensure complete histological and clinical remission of $\mathrm{CD}$, improving quality of life and reducing the risk of long-term complications in patients with gastrointestinal symptoms, as well as in asymptomatic patients. Poor dietary compliance has proved to be correlated with fertility problems, osteopenia, an increased risk of bone fractures, and the onset of other autoimmune diseases and lymphoma [3]. Although strict dietary compliance is fundamental for the health of celiac patients, there are no evidence-based recommendations on the best way to assess dietary compliance or on monitoring frequency [4]. Dietary compliance is typically assessed on the basis of dietary history, symptoms (if any), serum antibody tests and repeated small intestine biopsy. However, these methods have certain limitations.

A detailed dietary history, aimed at checking that foods with even minute traces of gluten are not ingested, is timeconsuming and calls for collaboration with a dietitian. Doctors therefore usually assess dietary compliance using structured questionnaires that investigate the quality and frequency of foods consumed. The fastest and most widely used is the Biagi questionnaire [5,6], based on 4 simple questions and yields a point score that has proven sufficiently reliable, showing a good correlation with histology. However, a limit of this and other questionnaires is that they assume that patients know exactly the gluten content of what they eat and that they may report their degree of compliance incorrectly because of unintentional mistakes [7]. Another tool is evaluation of symptoms, but again it is not completely reliable because many celiacs have atypical symptoms or are asymptomatic. Duodenal biopsy is considered the gold standard for the diagnosis of CD [8] and for assessment of dietary compliance, but it is an expensive and invasive procedure unsuited for frequent disease monitoring.

With regard to serum antibody tests, it has been observed that serum parameters, especially anti-tissue transglutaminase (tTG) IgA antibodies [9], become negative 1 or 2 years after starting a GFD. However, various studies have shown that in CD patients on GFD, tTG IgA values are not always correlated with mucosal damage [10] and in any case are not sufficiently sensitive to detect occasional dietary transgressions that could prevent histological recovery $[11,12]$.

Since all of the methods used so far fail to offer a completely reliable measure of dietary compliance, or are impractical, it was recently proposed to assay gluten immunogenic peptides (GIPs), detectable in feces as a result of the incomplete breakdown of gluten in the gastrointestinal tract. If a GFD is followed strictly, there are no fecal GIPs, while on the other hand when they can be detected it means that there has been gluten intake. Preliminary studies have shown that GIPs can be assayed with a sensitivity of $98.5 \%$ and a specificity of $100 \%[7,13]$. For this reason, detection of fecal GIPs has been proposed as an effective, noninvasive method of assessing the dietary compliance of celiac patients [14,15].

The aim of the present study was: 1) to confirm that measurement of GIPs in feces can be used as an indicator of dietary compliance in pediatric and adult CD patients; and
2) to compare this new marker with traditional methods of evaluating GFD compliance.

\section{Patients and methods}

\section{Enrolment of subjects and collection of samples}

From March 2018 to February 2019, we enrolled 55 consecutive CD patients (27 adults and 28 children, age 6-72 years) who had been on a GFD for at least 2 years. The patients were monitored at the Gastroenterology \& Endoscopy and Pediatric Units of Siena University Hospital. Patients and parents of minors gave their written informed consent to participation in the study. We excluded patients who had suffered episodes of diarrhea in the week when biological samples were obtained and those judged insufficiently reliable, as well as those in situations that could have caused dietary non-compliance (e.g., a recent school trip for children; business travel and frequent meals at restaurants for adults).

Diagnosis of CD was based on the criteria of the European Society for Pediatric Gastroenterology, Hepatology and Nutrition (ESPGHAN) [16] for children and on the guidelines of the American College of Gastroenterology [17] for adults. All patients were evaluated clinically for symptoms, physical parameters (body weight, height, body mass index [BMI]) and laboratory parameters (total blood count, iron, ferritin, calcium, and alanine and aspartate aminotransferase). AntitTG IgA antibodies were measured by fluoroimmunoenzyme assay (Thermofisher, Uppsala, Sweden). According to the manufacturer, values $<7 \mathrm{U} / \mathrm{mL}$ are negative, $7-10 \mathrm{U} / \mathrm{mL}$ are borderline and $>10 \mathrm{U} / \mathrm{mL}$ are positive. For the purposes of this study, borderline values were considered negative.

Compliance with GFD was evaluated using the Biagi questionnaire [6], in which scores of 0 or 1 indicate that the patient is not on a strict GFD, a score of 2 indicates that the patient follows the diet but makes mistakes that need to be corrected, and scores of 3 or 4 indicate that the patient follows the diet strictly. The study was approved by the Tuscan Regional Ethics Committee for Clinical Experimentation, South-East branch (Protocol no. 12725-2018).

\section{Extraction of fecal GIPs}

Feces were sampled with the GIP Coprocollect device (DIESSE Diagnostica Senese, Siena, Italy), which consists of a tube with a cap connected to a stick ending with a spoon. The device has a label, indicating the tare weight, so that the exact quantity of sample can be determined. Samples were frozen and stored at $-20^{\circ} \mathrm{C}$ until processing.

Only one commercial sandwich enzyme-linked immunosorbent (ELISA) assay is currently available for detection of GIPs in feces: the iVYLISA GIP-S test (Biomedal S.L., Seville, Spain). This test uses the G12 anti-33mer 
monoclonal antibody and can detect GIPs/g feces in the nanogram range. Despite its high performance, it involves a complex extraction step for fecal GIPs, with incubation at $50^{\circ} \mathrm{C}$ for $60 \mathrm{~min}$ and manual mixing of the sample for 10 sec every 15 min. Diesse introduced modifications to simplify and improve the extraction procedure: the extraction solution, added on the basis of the amount of sample, enables extraction of GIPs from the primary sample without hand mixing. The small magnet fitted to the device ensures better shaking and extraction is conducted at room temperature.

\section{Assay of fecal GIPs}

After extraction, fecal concentrations of GIPs were determined by the iVYLISA GIP-S test on a Chorus analyzer (DIESSE), an automated random-access instrument for immunometric assay with ready-to-use single-test devices. Chorus software was modified for automated GIP testing so as to obtain results that could be compared with those from the manual method. No cutoff is provided by the manufacturer, since GIPs should be undetectable in the absence of gluten ingestion. The limit of detection is $156 \mathrm{ng} / \mathrm{g}$ feces and values below this concentration are considered negative.

\section{Statistical analysis}

To meet the first aim of the study, we evaluated the prevalence of positivity for fecal GIPs and its 95\% confidence interval (CI). The characteristics of patients testing positive for fecal GIPs were compared with those of patients testing negative using descriptive statistical methods. Estimating $80 \%$ of GIP negatives in the low compliance group and $20 \%$ in the high compliance group and applying the Fisher exact test for an effect size of 0.6 , a type 1 error $\alpha$ of 0.05 and a power $1-\beta$ of 0.95 , we obtained a minimum sample size of 50 cases. Categorical variables were compared using the one-tailed Fisher exact test and the chi-squared test, whereas continuous variables were compared using the Mann-Whitney and Student's $t$-tests (2-tailed), since the variables under consideration were normally distributed. To obtain the predictive power of certain measures with respect to the dependent variable described by compliance with GFD, we created a series of logistic regression models with the following independent variables: presence of symptoms, patient group (adults vs. children), tTG IgA antibodies, Biagi score (dichotomized as: no GFD or GFD with mistakes, vs. strict GFD) and positivity for fecal GIPs. Only variables that made a significant contribution to the logistic regression model were included in the final model. The model assumptions were checked and fit was assessed using Nagelkerke's R-squared and the Hosmer-Lemeshow test. The contribution of the predictors to the final binary logistic regression model was analyzed using the Wald test. For analysis of data we used IBM SPSS v25 software, setting significance at $\mathrm{P}<0.05$.

\section{Results}

Eight patients (14.5\%; 95\% CI: 5.19-23.81; 2 adults and 6 children) tested positive for fecal GIPs (GIP+) and 47 patients (85.5\%; 25 adults and 22 children) tested negative (GIP-). In positive subjects, GIPs values ranged from 180 to $685.9 \mathrm{ng} / \mathrm{g}$ feces. The demographic and clinical characteristics of GIPs+ and GIPs- subjects are shown in Table 1. The proportion of male and female patients was similar for GIPs+ and GIPs$(\mathrm{P}=0.67)$.

The percentage of asymptomatic GIP+ patients was $71.4 \%$. The percentage of symptomatic GIP+ patients was lower than the percentage of symptomatic GIP- patients (28.6\% vs. $35 \%)$. Patients in the GIP+ group had a lower mean age that patients in the GIP- group $(22 \pm 10.65$ years vs. $29.89 \pm 19.22)$ and also had a lower mean age at diagnosis $(13.67 \pm 12.75$ years vs. $24.64 \pm 20.59)$. The GIP+ group also had a longer history of CD (111.5 \pm 65.18 years vs. $71.6 \pm 63.8$ months), which coincided with the duration of GFD. Height was slightly greater in the $\mathrm{GIP}+$ group, whereas body weight and BMI were similar. The results of laboratory tests did not differ between the 2 GIP groups (Table 2).

Anti-tTG IgA antibodies were found in 3/8 GIP+ patients and in 3/47 GIP- patients. In the latter group, antibody values were all less than 1.5 times the cutoff value. The difference between the 2 groups was not significant $(\mathrm{P}=0.4)$.

The Biagi score was evaluated as a dichotomous variable, dividing patients into 2 groups: patients not on a GFD or who make mistakes (score 0-2) and patients complying strictly with a GFD (score 3-4). According to the Biagi score, 94.6\% of GIPpatients showed strict dietary compliance, whereas $42.9 \%$ of GIP+ patients were not on a GFD or made substantial mistakes (Table 3). The Biagi score showed a significant association with positivity for fecal GIPs $(\mathrm{P}=0.02)$. However, according to this score, $57.1 \%$ of GIP+ patients proved to show strict dietary compliance and $5.4 \%$ of GIP- patients were not on a GFD or made substantial mistakes.

With regard to the predictive capacity of the following parameters for strict compliance with GFD: patient group (adults vs. children), presence/absence of symptoms, positivity for anti-tTG IgA antibodies, Biagi score and positivity for fecal GIPs, the binary logistic regression model performed with single predictors showed that only positivity for fecal GIPs $(\mathrm{P}=0.04)$ and Biagi score $(\mathrm{P}=0.02)$ contributed significantly to the detection of dietary compliance. These 2 variables were included in the final model, which is illustrated in Table 4. The high value of Nagelkerke R-squared (0.461) and the lack of significance shown by the Hosmer-Lemeshow test $(\mathrm{P}=0.85)$ demonstrated the good fit of the model to the data. Both parameters showed a negative sign, indicating that absence of GFD or making substantial errors $(\beta=-2.718)$ and positivity for fecal GIPs $(\beta=-2.555)$ reduced the level of compliance with GFD, an effect that was slightly higher for GIP positivity (Wald's chi-square $=5.450$ ) than for the Biagi score (Wald's chisquare $=4.001$ ). 
Table 1 Characteristics of subjects positive and negative for fecal gluten immunogenic peptides (GIPs)

\begin{tabular}{|c|c|c|c|c|}
\hline Characteristics & & GIP-positive subjects & GIP-negative subjects & P-value \\
\hline \multirow[t]{2}{*}{ Sex } & Female & $6(75.0 \%)$ & $35(74.5 \%)$ & 0.67 \\
\hline & Male & $2(25.0 \%)$ & $12(25.5 \%)$ & \\
\hline \multirow[t]{2}{*}{ Symptoms } & No & $5(71.4 \%)$ & $26(65 \%)$ & 0.55 \\
\hline & Yes & $2(28.6 \%)$ & $14(35 \%)$ & \\
\hline \multirow[t]{3}{*}{ Marsh-Oberhuber at diagnosis } & 2 & $0(0.0 \%)$ & $2(5.4 \%)$ & 0.66 \\
\hline & 3 & $2(40.0 \%)$ & $20(54.1 \%)$ & \\
\hline & $3 \mathrm{~A} / \mathrm{B} / \mathrm{C}$ & $3(60.0 \%)$ & $15(40.5 \%)$ & \\
\hline Age $($ mean $\pm S D)$ & & $22.0 \pm 10.6$ & $29.8 \pm 19.2$ & 0.48 \\
\hline Age $($ mean \pm SD) at diagnosis & & $13.6 \pm 12.7$ & $24.6 \pm 20.5$ & 0.28 \\
\hline Duration (mean $\pm \mathrm{SD}$ ) of disease (months) & & $111.5 \pm 65.1$ & $71.6 \pm 63.8$ & 0.12 \\
\hline Duration (mean $\pm \mathrm{SD}$ ) of gluten-free diet (months) & & $111.5 \pm 65.1$ & $71.0 \pm 65.3$ & 0.12 \\
\hline Body weight (mean \pm SD) $(\mathrm{kg})$ & & $53.6 \pm 11.1$ & $53.6 \pm 16.1$ & 0.82 \\
\hline Height (mean $\pm \mathrm{SD})(\mathrm{cm})$ & & $162.7 \pm 11.2$ & $158.1 \pm 16.5$ & 0.54 \\
\hline $\mathrm{BMI}($ mean $\pm \mathrm{SD})$ & & $20.1 \pm 2.9$ & $20.5 \pm 3.5$ & 0.65 \\
\hline
\end{tabular}

SD, standard deviation; BMI, body mass index

Table 2 Association between laboratory parameters and positivity/ negativity for fecal gluten immunogenic peptides (GIPs)

\begin{tabular}{lccc}
\hline Parameters & $\begin{array}{c}\text { GIPs positive } \\
\text { median }\end{array}$ & $\begin{array}{c}\text { GIPs negative } \\
\text { median }\end{array}$ & P-value \\
\hline $\begin{array}{l}\text { Hemoglobin } \\
\text { Hematocrit }\end{array}$ & 14.3 & 12.7 & 0,48 \\
$\begin{array}{l}\text { Mean corpuscular } \\
\text { volume }\end{array}$ & 42.9 & 39.7 & 0.43 \\
$\begin{array}{l}\text { Red cell } \\
\text { distribution width }\end{array}$ & 13.3 & 85.8 & 0.49 \\
$\begin{array}{l}\text { Alanine } \\
\text { aminotransferase }\end{array}$ & 15 & 13.3 & 0.76 \\
$\begin{array}{l}\text { Aspartate } \\
\text { aminotransferase }\end{array}$ & 21 & 165 & 0.44 \\
Iron & 92 & 106.5 & 0.69 \\
Ferritin & 29 & 33 & 0.97 \\
Calcium & 9.3 & 9.4 & 0.90 \\
\hline
\end{tabular}

Table 3 Association between compliance with a gluten-free diet (GFD), evaluated by Biagi score and positivity/negativity for fecal gluten immunogenic peptides (GIPs)

\begin{tabular}{lccc}
\hline Biagi score & $\begin{array}{c}\text { GIPs } \\
\text { positive }\end{array}$ & $\begin{array}{c}\text { GIPs } \\
\text { negative }\end{array}$ & Total \\
\hline $\begin{array}{l}\text { Non-compliance with GFD or } \\
\text { GFD with major errors }\end{array}$ & 3 & $2(5.4 \%)$ & 5 \\
Strict GFD & 4 & 35 & $(11.4 \%)$ \\
Total & $(57.1 \%)$ & $(94.6 \%)$ & $(88,6 \%)$ \\
\hline
\end{tabular}

\section{Discussion}

The current routine approach to ascertaining dietary compliance is an overall assessment of $\mathrm{CD}$ patients that includes food history, structured questionnaires, clinical symptoms, assay of tTG IgA antibodies and duodenal biopsy for difficult cases [18]. In this study we verified the utility of a new automated test involving the determination of GIPs in feces in order to evaluate compliance with a GFD. Since GIPs are only excreted in feces in the case of gluten intake, their detection in CD patients on a GFD means that there has been accidental exposure or voluntary laxity with the diet. The results showed that $14.5 \%$ of the celiac patients analyzed, who had been on GFD for at least 2 years, showed detectable quantities of GIPs in the feces.

The most interesting finding is that, although we found a significant association between Biagi score and positivity for fecal GIPs (confirming our preliminary data [19]), 57.1\% of GIP+ patients declared that they had not consumed any gluten and were therefore not identified by the food questionnaire. These results confirm the limits of questionnaires: because they are not based on objective data but on the patient's perceptions, they cannot identify voluntary or accidental dietary transgressions. Patients may purposely not admit gluten intake, may not know the exact gluten content of what they consume, or may eat foods unaware that they contain gluten.

With regard to assessment of symptoms, follow up on initial or new symptoms is useful to check improvement and the course of CD, but many celiacs are asymptomatic or minimally symptomatic at presentation and while on a GFD. A controlled study of the effects of gluten challenge found that symptoms 
Table 4 Parameters of the final binary logistic regression model with compliance with gluten-free diet (GFD) as dependent variable and Biagi score and positivity for fecal gluten immunogenic peptides (GIPs) as predictors

\begin{tabular}{lccccc}
\hline Parameters & $\beta$ & $\begin{array}{c}\text { Standard } \\
\text { error }\end{array}$ & Wald's chi-square & $\begin{array}{c}\text { Degrees of } \\
\text { freedom }\end{array}$ & $\begin{array}{c}\text { P-value } \\
\text { Positivity for fecal GIPs }\end{array}$ \\
Biagi score (No GFD or GFD with substantial mistakes) & -2.555 & 1.095 & 5.450 & 1 & 0.02 \\
Constant & 2.718 & 1.359 & 4.001 & 1 & 0.04 \\
\hline
\end{tabular}

were absent in $22 \%$ of celiacs, despite their showing significant evidence of villous atrophy when small intestine biopsy specimens underwent histological examination [20].

In our study, $66 \%$ of patients were asymptomatic and $16 \%$ of these tested positive for fecal GIPs. On the other hand, $34 \%$ of patients were symptomatic and $12.5 \%$ of these tested positive for fecal GIPs. These results confirm those of previous studies showing that $25-40 \%$ of adult celiacs after 2 years on GFD and 5-19\% of children after 1 year on GFD showed persistent enteropathy [21-23]. In this context, persistent gluten intake, due to conscious breaking of the diet or accidental exposure to small quantities of gluten, is one of the most frequent causes of symptoms [24], since only 1-2\% develop refractory CD [25]. Moreover, the fact that a higher percentage of asymptomatic than symptomatic patients are GIP+ suggests that there is a much lower likelihood of finding fecal GIPs in symptomatic than in asymptomatic patients. The desire to avoid symptoms is effectively the main reason for strict compliance with the diet [26]. The finding that $87.5 \%$ of symptomatic patients were GIP-negative is interesting: in these subjects symptoms could be the result of coexisting disorders, such as irritable bowel syndrome or microscopic colitis. Taken together, these findings confirm that it is not possible to use symptoms alone as an indicator of compliance with GFD and mucosal healing.

Serological testing is widely used in the routine monitoring of CD patients on GFD, although the results are not always correlated with histological results or symptoms. This could be due to the long half-life of antibodies and to the fact that antibody levels reflect immune response rather than direct intestinal damage. Anti-tTG IgA and IgG antibodies can often take many months (6-24 months) to decrease after the antigen source is eliminated from the diet, especially if the initial value is very high, and anti-tTG IgA antibodies generally return negative later in adults than in children. A meta-analysis of 11 studies covering 1088 patients estimated that the sensitivity of anti-tTG IgA antibodies was 50\% and the specificity for persisting villous atrophy in subjects without gluten intake was $83 \%$ [10]. In our study, $62.5 \%$ of GIP+ patients showed normal anti-tTG IgA antibody levels. These results are in line with data in the literature showing the low sensitivity of these serum markers in monitoring response to diet [7], and above all indicate that they are not suitable for detecting small or infrequent exposure to gluten [27]. Concentrations $>10 \mathrm{U} /$ $\mathrm{mL}$ of anti-tTG were only found in $37.5 \%$ of GIP+ patients. It can presumably be excluded that these values were due to persistence of antibodies. The subjects enrolled in the study had been on GFD for many years and, had they complied strictly, serum levels of anti-tTG IgA ought to have returned to normal. To the contrary, their anti-tTG IgA levels indicated repeated and not occasional gluten intake; this is consistent with a recent study by Comino et al [28] in which GIPs+ subjects showed serum concentrations of anti-tTG IgA $>10 \mathrm{U} /$ $\mathrm{mL}$ that persisted longer than in patients who tested negative to fecal GIPs.

Our hypothesis is also supported by the fact that we observed a statistically significant association $(\mathrm{P}=0.03)$ between anti-tTG IgA and Biagi score, so that those who according to the questionnaire were not on GFD showed a higher median value of anti-tTG IgA than those who followed it strictly (7.4 vs. $1.4 \mathrm{U} / \mathrm{mL})$, suggesting a behavioral model of repeated or chronic exposure to gluten, rather than episodic or rare exposure.

In the case of elevated values of anti-tTG IgA and negative GIPs, a duodenal biopsy may be indicated, given that only a repeatedly non-compliant diet leads to a histological lesion and causes an increase in anti-tTG levels, and that GIPs could be negative because gluten has not been consumed recently. Moreover, in view of the high sensitivity of GIPs, it may be useful to carry out endoscopy in patients who have repeatedly tested positive for GIPs, in order to detect intestinal damage induced by prolonged ingestion of even minimal quantities of gluten.

These observations raise the question of whether GIP positivity is correlated with the degree of intestinal atrophy. There is only one study in the literature that looked for correlations between GIPs and mucosal damage [12]. In that study, analysis of duodenal biopsies revealed that most patients with CD (89\%) without villous atrophy had no detectable GIPs in urine, while all patients with quantifiable GIPs in urine showed incomplete recovery of the intestinal mucosa. Though GIP testing was performed in urine, the same good correlation presumably also exists for fecal GIPs.

In our study, transgressions were mostly related to the phase in which children reach puberty and adolescence and have increasing independence in food choice: $75 \%$ of GIP+ patients were younger than GIP- patients; in particular, GIP+ pediatric patients not identified by the Biagi questionnaire were aged between 11 and 18 years, and were therefore in precisely that stage of development in which adolescents are most resistant to following GFD and are more likely to break the diet intentionally $[29,30]$. 
Older children and adolescents also eat more often away from home (at school, at the homes of friends) where the food is prepared by persons who are not always informed about the requirements of a GFD. The higher rate of positivity to GIPs in this age group may also be related to behaviors of this kind associated with a higher risk of exposure to gluten. Patients in the GIP+ group also showed a lower mean age at diagnosis, had a longer history of CD and had been longer on GFD than subjects negative for fecal GIPs. As was also observed in the study by Stefanolo et al [31], we found that positivity for GIPs increased with the time since diagnosis, and this may reflect a tendency to pay less attention to diet over time, especially in the case of asymptomatic subjects who begin to think they can tolerate small amounts of gluten and consequently adjust their diet.

We acknowledge that this study has some limitations. First, the lower rate of GIP-positive patients compared with other studies where multiple stool samples were collected may be due to differences in study design. Since fecal analysis of GIPs is an accurate method for assessing gluten exposure shortly after ingestion, it is likely that the single stool sample collected in our study might underestimate GIP positivity. Another limitation of our study concerns sample size, determined for the primary objective, but is probably too small for the other statistical tests we performed. These results must therefore be viewed with caution, since they could be affected by an uncontrolled type II error.

In conclusion, the determination of fecal GIPs proves to be an excellent method for checking dietary compliance. Unlike traditional methods that only assess the consequences of dietary transgressions, the detection of GIPs in feces enables a direct, noninvasive, objective, quantitative assessment of exposure to gluten, including occasional and minor exposure. The results of binary logistic regression analysis showed that 2 variables suited to assess compliance with GFD were the Biagi score and fecal GIPs. Their combined use would seem to be the best way to monitor dietary compliance, reducing delays, costs and the need for other tests, some of which are invasive. Moreover, the determination of fecal GIPs promises to be useful in the clinical management of $\mathrm{CD}$, making it possible to: check gluten intake at the time of diagnosis; document a substantial decrease in gluten in the first phase of GFD (since the first year is fundamental for restoring the normal structure of the intestinal mucosa); verify a patient's eating habits at any time during the follow up; check involuntary gluten intake; monitor celiacs in whom symptoms or positivity of serum biomarkers persist; and avoid unnecessary biopsies. The detection of GIPs in stools may also facilitate the identification of patients with refractory $\mathrm{CD}$, characterized by persistence of symptoms and villous atrophy despite strict compliance with GFD for $>12$ months. This test would rule out exposure to gluten or confirm dietary non-compliance, enabling better differential diagnosis. Analysis of fecal GIPs also promises to be useful in pharmacological research involving new drugs for $C D$, to check their efficacy to remove ingested gluten.

\section{Summary Box}

\section{What is already known:}

- A strict lifelong gluten-free diet is the only available treatment for celiac disease

- Current methods of ascertaining compliance to a gluten-free diet by celiac patients have certain limitations

- Detection of fecal gluten peptides has been proposed as an effective method of assessing dietary compliance of celiac patients

\section{What the new findings are:}

- Fecal gluten peptides were detected in $14.5 \%$ of celiac patients who had been on a gluten-free diet for at least 2 years

- Assay of fecal gluten peptides identified more patients who did not comply with the diet than did the Biagi questionnaire, evaluation of symptoms or detection of anti-tissue transglutaminase IgA

- The combined use of the Biagi score and fecal gluten peptides would seem to be the best way to monitor dietary compliance, reducing the need for other tests

\section{References}

1. Ludvigsson JF, Leffler DA, Bai JC, et al. The Oslo definitions for coeliac disease and related terms. Gut 2013;62:43-52.

2. Morón B, Bethune MT, Comino I, et al. Toward the assessment of food toxicity for celiac patients: characterization of monoclonal antibodies to a main immunogenic gluten peptide. PLoS One 2008; 3:e2294.

3. Lebwohl B, Granath F, Ekbom A, et al. Mucosal healing and mortality in coeliac disease. Aliment Pharmacol Ther 2013;37:332-339.

4. Bai JC, Fried M, Corazza GR, et al; World Gastroenterology Organization. World Gastroenterology Organisation global guidelines on celiac disease. J Clin Gastroenterol 2013;47:121-126.

5. Biagi F, Andrealli A, Bianchi PI, Marchese A, Klersy C, Corazza GR. A gluten-free diet score to evaluate dietary compliance in patients with coeliac disease. Br J Nutr 2009;102:882-887.

6. Biagi F, Bianchi PI, Marchese A, et al. A score that verifies adherence to a gluten-free diet: a cross-sectional, multicentre validation in real clinical life. Br J Nutr 2012;108:1884-1888.

7. Comino I, Fernández-Bañares F, Esteve $M$, et al. Fecal gluten peptides reveal limitations of serological tests and food questionnaires for monitoring gluten-free diet in celiac disease patients. Am J Gastroenterol 2016;111:1456-1465.

8. Leonard MM, Weir DC, DeGroote M, et al. Value of IgA tTG in predicting mucosal recovery in children with celiac disease on a gluten-free diet. J Pediatr Gastroenterol Nutr 2017;64:286-291.

9. Porcelli B, Alessio MG, Villalta D, et al. Guidelines for laboratory and histological diagnosis of celiac disease. Revision 2015. Riv It 
Med Lab 2015;11:76-95.

10. Silvester JA, Kurada S, Szwajcer A, Kelly CP, Leffler DA, Duerksen DR. Tests for serum transglutaminase and endomysial antibodies do not detect most patients with celiac disease and persistent villous atrophy on gluten-free diets: a meta-analysis. Gastroenterology 2017;153:689-701.

11. VahediK, MascartF,MaryJY,etal. Reliability of antitransglutaminase antibodies as predictors of gluten-free diet compliance in adult celiac disease. Am J Gastroenterol 2003;98:1079-1087.

12. Moreno ML, Cebolla, Muñoz-Suano A, et al. Detection of gluten immunogenic peptides in the urine of patients with coeliac disease reveals transgressions in the gluten-free diet and incomplete mucosal healing. Gut 2017;66:250-257.

13. Roca M, Donat E, Masip E, et al. Detection and quantification of gluten immunogenic peptides in feces of infants and their relationship with diet. Rev Esp Enferm Dig 2019;111:106-110.

14. Comino I, Real A, Vivas S, et al. Monitoring of gluten-free diet compliance in celiac patients by assessment of gliadin 33-mer equivalent epitopes in feces. Am J Clin Nutr 2012;95:670-677.

15. Moreno ML, Rodríguez-Herrera A, Sousa C, Comino I. Biomarkers to monitor gluten-free diet compliance in celiac patients. Nutrients 2017;9:46.

16. Husby S, Koletzko S, Korponay-Szabó IR, et al; European Society for Pediatric Gastroenterology, Hepatology, and Nutrition. European Society for Pediatric Gastroenterology, Hepatology, and Nutrition guidelines for the diagnosis of coeliac disease. J Pediatr Gastroenterol Nutr 2012;54:136-160.

17. Rubio-Tapia A, Hill ID, Kelly CP, Calderwood AH, Murray JA; American College of Gastroenterology. ACG clinical guidelines: diagnosis and management of celiac disease. Am J Gastroenterol 2013;108:656-676.

18. Rodrigo L, Pérez-Martinez I, Lauret-Braña E, Suárez-González A. Descriptive study of the different tools used to evaluate the adherence to a gluten-free diet in celiac disease patients. Nutrients 2018;10:1777.

19. Porcelli B, Ferretti F, Cinci F, et al. Fecal gluten immunogenic peptides as indicators of dietary compliance in celiac patients. Minerva Gastroenterol Dietol 2020;66:201-207.
20. Lähdeaho ML, Mäki M, Laurila K, Huhtala H, Kaukinen K. Small- bowel mucosal changes and antibody responses after low- and moderate-dose gluten challenge in celiac disease. $B M C$ Gastroenterol 2011;11:129.

21. Ludvigsson JF, Murray JA. Epidemiology of celiac disease. Gastroenterol Clin North Am 2019;48:1-18.

22. Mahadev S, Murray JA, Wu TT, et al. Factors associated with villus atrophy in symptomatic coeliac disease patients on a gluten-free diet. Aliment Pharmacol Ther 2017;45:1084-1093.

23. Leonard MM, Fasano A. Zero, one, or two endoscopies to diagnose and monitor paediatric celiac disease? The jury is still out. J Pediatr Gastroenterol Nutr 2017;65:270-271.

24. Bai JC, Ciacci C. World Gastroenterology Organisation Global Guidelines: Celiac Disease February 2017. J Clin Gastroenterol 2017;51:755-768.

25. Leffler DA, Dennis M, Hyett B, Kelly E, Schuppan D, Kelly CP. Etiologies and predictors of diagnosis in nonresponsive celiac disease. Clin Gastroenterol Hepatol 2007;5:445-450.

26. Sansotta N, Amirikian K, Guandalini S, Jericho H. Celiac disease symptom resolution: effectiveness of the gluten-free diet. J Pediatr Gastroenterol Nutr 2018;66:48-52.

27. Rashid M, Lee J. Serologic testing in celiac disease. Can Fam Physician 2016;62:38-43.

28. Comino I, Segura V, Ortigosa L, et al. Prospective longitudinal study: use of faecal gluten immunogenic peptides to monitor children diagnosed with coeliac disease during transition to a gluten-free diet. Aliment Pharmacol Ther 2019;49:1484-1492.

29. Hall NJ, Rubin G, Charnock A. Systematic review: adherence to a gluten-free diet in adult patients with coeliac disease. Aliment Pharmacol Ther 2009;30:315-330.

30. White LE, Bannerman E, Gillett PM. Coeliac disease and the gluten-free diet: a review of the burdens; factors associated with adherence and impact on health-related quality of life, with specific focus on adolescence. J Hum Nutr Diet 2016;29:593-606.

31. Stefanolo JP, Talamo M, Dodds S, et al. Real-world gluten exposure in patients with celiac disease on gluten-free diet, determined from gliadin immunogenic peptides in urine and fecal samples. Clin Gastroenterol Hepatol 2020 Mar 23 [Online ahead of print]. doi: 10.1016/j.cgh.2020.03.038. 